\title{
Ultrasmall Dual-Band Metamaterial Antennas Based on Asymmetrical Hybrid Resonators
}

\author{
Ji-Xu Zhu, Peng Bai, and Jia-Fu Wang \\ Equipment Management and Safety Engineering College, Air Force Engineering University, Xian 710051, China \\ Correspondence should be addressed to Ji-Xu Zhu; zhujixu601@126.com
}

Received 18 April 2016; Revised 15 June 2016; Accepted 16 June 2016

Academic Editor: Jaume Anguera

Copyright (c) $2016 \mathrm{Ji}-\mathrm{Xu}$ Zhu et al. This is an open access article distributed under the Creative Commons Attribution License, which permits unrestricted use, distribution, and reproduction in any medium, provided the original work is properly cited.

\begin{abstract}
A new type of hybrid resonant circuit model is investigated theoretically and experimentally. The resonant model consists of a right hand (RH) patch part and a composite right and left handed (CRLH) part (RH + CRLH), which determines a compact size and also a convenient frequency modulation characteristic for the proposed antennas. For experimental demonstration, two antennas are fabricated. The former dual-band antenna operating at $f_{-1}=3.5 \mathrm{GHz}$ (Wimax) and $f_{+1}=5.25 \mathrm{GHz}$ (WLAN) occupies an area of $0.21 \lambda_{0} \times 0.08 \lambda_{0}$, and two dipolar radiation patterns are obtained with comparable gains of about 6.1 and $6.2 \mathrm{~dB}$, respectively. The latter antenna advances in many aspects such as an ultrasmall size of only $0.16 \lambda_{0} \times 0.08 \lambda_{0}$, versatile radiation patterns with a monopolar pattern at $f_{0}=2.4 \mathrm{GHz}$ (Bluetooth), and a dipole one at $f_{+1}=3.5 \mathrm{GHz}$ (Wimax) and also comparable antenna gains. Circuit parameters are extracted and researched. Excellent performances of the antennas based on hybrid resonators predict promising applications in multifunction wireless communication systems.
\end{abstract}

\section{Introduction}

Over the past decade, artificial electromagnetic (EM) metamaterials (MTMs) have intrigued long-hold interests due to their abundant abnormal behaviors that are not occurring in nature. As a result, scientists and engineers have endeavored theoretically and experimentally to bring these special MTMs into applications such as microwave devices [1] and new functional devices [2]. As an important part for wireless communication system, antennas based on MTMs have developed rapidly with enhanced performances such as high directivity, multibeams, multifrequency, and broad bandwidth [3-7].

Resonant antennas by loading MTMs are interesting for researchers for their compact size and multibands characteristic, among which two categories are most important. Firstly, resonant antennas using composite right and left handed (CRLH) transmission line (TL) are in advantage of compact structure, while they suffer from an easy method of frequency modulation $[8,9]$. For another, hybrid resonant multifrequency antennas with a symmetrical structure of right handed patch, CRLH part, and right handed patch (RH + CRLH + RH) are capable of frequency modulation [10-16], while they are not sufficient in miniaturization.
To solve the above issues, in this paper, a new kind of CRLH TLs has been proposed by loading complementary spiral resonators (CSRs) in the conventional mushroom structure. And a novel asymmetrical hybrid resonant circuit model is investigated theoretically for the first time in Section 2. For verification and experimental applications, two dual-band dual-mode antennas are designed, fabricated, and measured. In Section 3, a patch antenna filled with 1 $\times 2$ conventional mushroom structures is investigated. In Section 4, dual-band ultrasmall antenna by loading CSRs for further miniaturization and easy frequency modulation is studied. Circuit parameters are extracted for deep insight into the working mechanism of the hybrid resonant circuit model. Finally, a conclusion of the whole paper is made.

\section{CRLH Cell Design and the Working Mechanism of Novel Asymmetrical Hybrid Resonator}

To date, various types of CRLH TLs have been investigated since the first proposal. Metallic via holes, as the most important structure to provide $\mathrm{LH}$ inductor, have been applied to design CRLH TLs, especially for the mushroom 




(a)

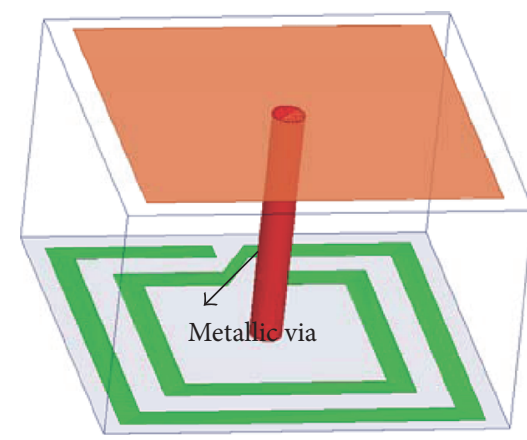

(b)

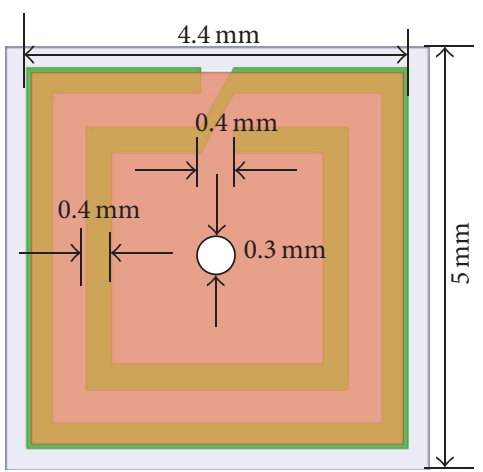

(c)

FIgURE 1: Topology of the conventional mushroom and CSRs-loaded mushroom structures. Perspective of (a) conventional mushroom cell and (b) CSRs-loaded mushroom element; (c) top and bottom view of the CSRs-loaded mushroom unit as well as geometrical dimensions.

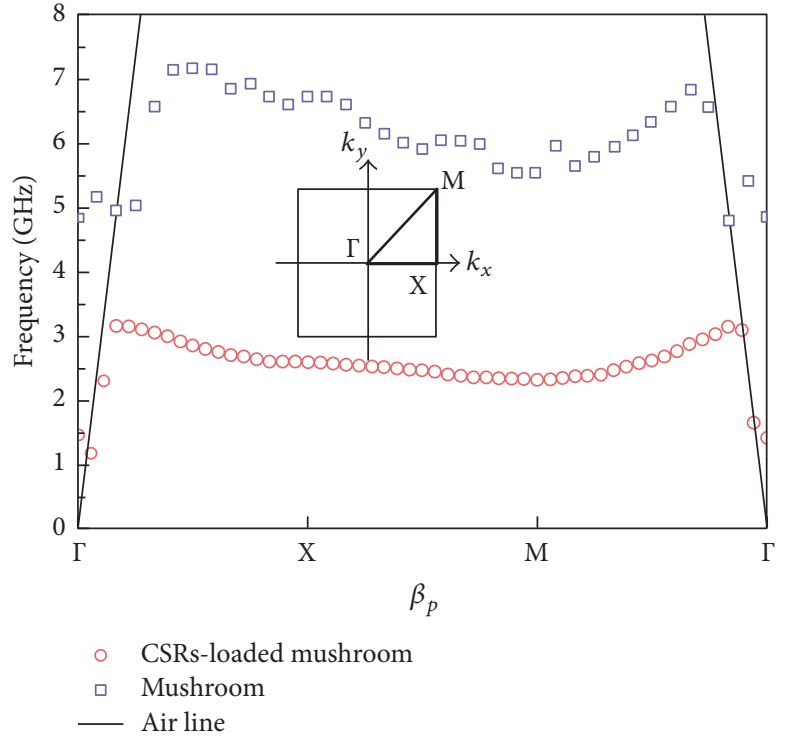

(a)

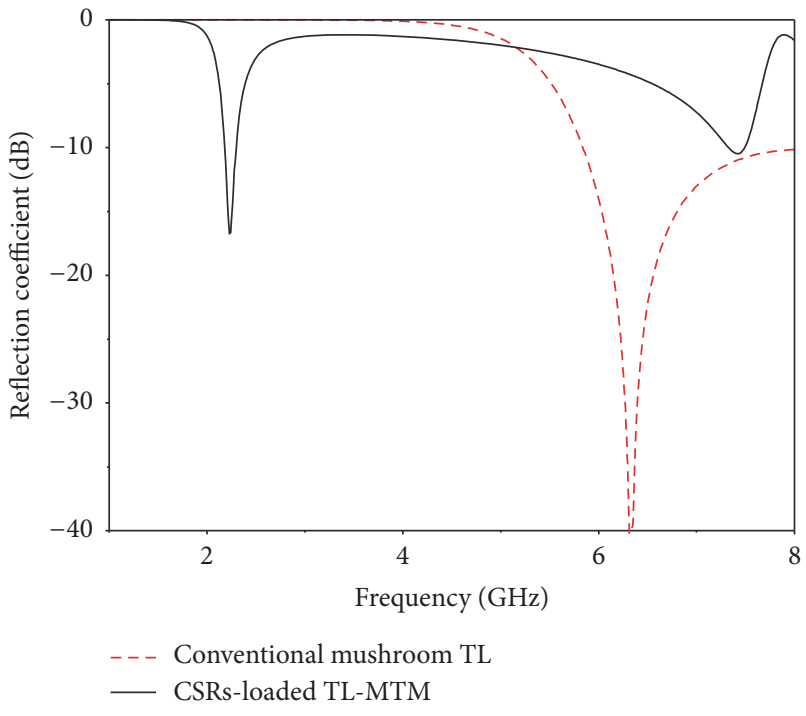

(b)

FIGURE 2: Dispersion diagram and simulated S-parameters of the conventional mushroom and CSRs-loaded mushroom TL structures.

CRLH TL (short for mushroom). In order to design an electrically smaller MTM and make a progress toward homogenized medium [17], a combination of two turns CSRs and mushroom structure is considered and optimized. For comparison, the conventional mushroom cell and the CSRsloaded mushroom cell are shown in Figure 1.

For design and analysis, the proposed mushroom TL cell and the upcoming antenna design are investigated on the $\mathrm{F} 4 \mathrm{~B}$ substrate with a dielectric constant of $\delta=2.65$ and a thickness of $h=3 \mathrm{~mm}$. The finite element method (FEM) based commercial software Ansoft HFSS is employed for full-wave EM simulation and Ansoft Serenade for electrical simulation. To gain a better understanding of the principle of the proposed CRLH TL, the dispersion curve is calculated and simulated using HFSS. Figure 2(a) compares the simulated dispersion diagram of the conventional mushroom and the CSRs-loaded mushroom structures. It is worth noting that the LH characteristic is clearly observed in the region of 5.51$7.25 \mathrm{GHz}$ for the conventional mushroom structure, whereas it dramatically shifts downward to $2.15-3.30 \mathrm{GHz}$ for the CSRs-loaded mushroom element. The reflection coefficients are also simulated for a complementary research with the results shown in Figure 2(b). It is obvious that the resonant frequency is about $6.5 \mathrm{GHz}$ for the conventional mushroom cell and $2.2 \mathrm{GHz}$ for the CSRs-loaded one, which further demonstrated the miniaturization by loading the CSRs perturbation. The increased LH inductor by etching the CSRs in the ground plane gives rise to a resonant frequency reduction of $66.15 \%$. And it may be an effective method to design electronic smaller antenna by loading the CSRs structure.

For a symmetrical $\mathrm{RH}+\mathrm{CRLH}+\mathrm{RH}$ resonator, two $\mathrm{RH}$ patches are essential for frequency manipulation [1016]. However, for an electrically small CRLH resonator, frequency modulation is not convenient $[8,9]$. So a novel 


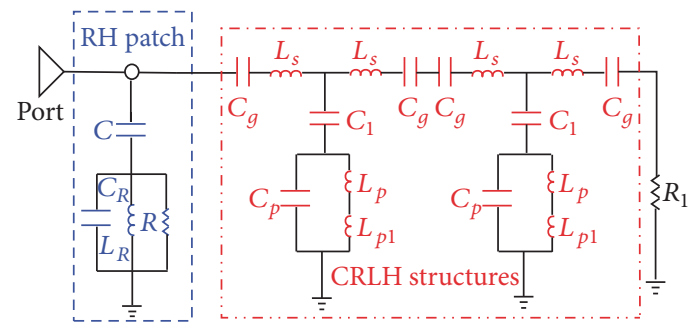

FIGURE 3: Illustration of the hybrid resonant equivalent circuit model.

asymmetrical hybrid resonator consisting of $\mathrm{RH}+\mathrm{CRLH}$ is concerned and investigated. The equivalent circuit model is shown in Figure 3. As expected, the final circuit model is actually a combination of $\mathrm{RH}$ patch part and CRLH part; for convenience and not losing universality, two CRLH cells are chosen. Completely different from conventional hybrid resonator, only one $\mathrm{RH}$ part is reserved aiming at both miniaturization and frequency manipulation. In the circuit model, the letter $C$ represents the coupling between the $\mathrm{RH}$ part and CRLH section. The EM response of the $\mathrm{RH}$ patch is modeled by the parallel resonant tank formed by $C_{R}$ and $L_{R}$, and $R$ represents the radiation resistance. For the CRLH parts, the left handed ( $\mathrm{LH}$ ) capacitor $C_{g}$ is provided by the slot coupling between adjacent cells. LH inductor $L_{p}$ is acquired by the metallic via hole connected to the ground, and $L_{p 1}$ is added for the frequency response of CSRs inserted in the mushroom structure. $L_{s}$ and $C_{p}$ is the inherent inductance and capacitance. $C_{1}$ accounts for the fringing capacitance of the gap. $R_{1}$ is set very large to simulate the condition of open circuit.

According to the circuit model in Figure 3, the dispersion relation for the novel resonant circuit model is as follows:

$$
\beta_{n} L=\beta_{n}^{\mathrm{RH}} d+\beta_{n}^{\mathrm{CRLH}} p N
$$

where the length of RH patch is defined as $d$ and $\beta_{n}^{\mathrm{RH}}$ is phase constant of the $\mathrm{RH}$ patch and can be calculated as:

$$
\beta_{n}^{\mathrm{RH}}=w \sqrt{L_{R} C_{R}}
$$

The number and propagation constant of the CRLH TL cells are defined as $N$ and $\beta_{n}^{\text {CRLH }}$ decided as

$$
\begin{aligned}
& \beta_{n}^{\mathrm{CRLH}} p \\
& \quad=\cos ^{-1}\left(1-0.5\left(\frac{w_{L}^{2}}{w^{2}}+\frac{w^{2}}{w_{R}^{2}}-\frac{w_{L}^{2}}{w_{s e}^{2}}-\frac{w_{L}^{2}}{w_{s h}^{2}}\right)\right)
\end{aligned}
$$

where $w_{L}, w_{R}, w_{s e}$, and $w_{s h}$ can be calculated as

$$
\begin{aligned}
w_{L} & =\frac{1}{\sqrt{C_{g}\left(L_{p}+L_{p 1}\right)}}, \\
w_{R} & =\frac{1}{\sqrt{C_{p} L_{s}}}, \\
w_{s e} & =\frac{1}{\sqrt{C_{g} L_{s}}}, \\
w_{s h} & =\frac{1}{\sqrt{\left(C_{p}+C_{1}\right)\left(L_{p}+L_{p 1}\right)}} .
\end{aligned}
$$

Then the resonant modes $n$ can be obtained by the following condition:

$$
\begin{aligned}
& \beta_{n} L=n \pi \\
& \quad(n=-N+1,-N+2, \ldots, 0,1,2, \ldots, N-2, N-1) .
\end{aligned}
$$

According to (1)-(5), two aspects should be highlighted. First, the resonant frequency is determined by both the $\mathrm{RH}$ patch and CRLH sections as the conventional hybrid resonator. Then, size reduction is available for a missing of $\mathrm{RH}$ patch and frequency modulation characteristic does not deteriorate with a combination of $\mathrm{RH}$ part and CRLH section.

\section{Dual-Band Antenna Design Using Hybrid Resonant Circuit Model}

According to the principle of the proposed hybrid resonator, a compact dual-band antenna shown in Figure 4 operating at $f_{-1}=3.5 \mathrm{GHz}$ (Wimax band) in $n=-1$ mode and $f_{+1}=5.25 \mathrm{GHz}$ (WLAN band) in $n=+1$ mode is designed by optimizing the RH patch and mushroom cells cautiously. As shown in Figure 4, the novel antenna consists of a metal patch and $1 \times 2$ conventional mushroom cells. The whole dimension of the antenna is just $17.8 \mathrm{~mm} \times 10 \mathrm{~mm}$, evaluated as $0.21 \lambda_{0} \times 0.08 \lambda_{0}$. It is promising that the proposed antenna is $90.31 \%$ smaller than conventional half wavelength antenna operating at $3.5 \mathrm{GHz}$, indicating a desirable performance in recent higher integration circuit.

Impedance match is essential to the radiation efficiency of an antenna, especially for dual-band or multiband antenna 




FIGURE 4: Perspective view of the proposed dual-band antenna with geometrical parameters (in mm).

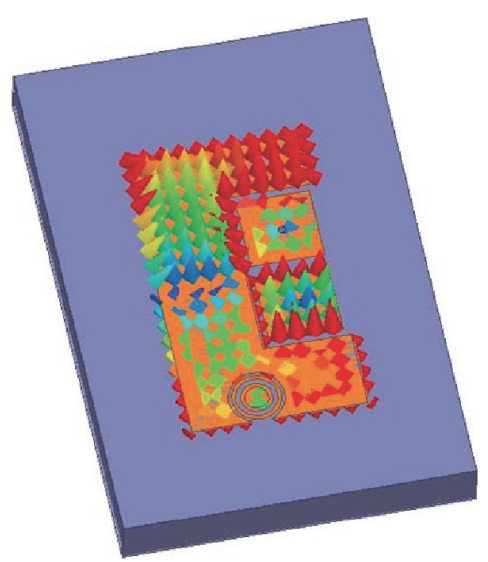

(a)

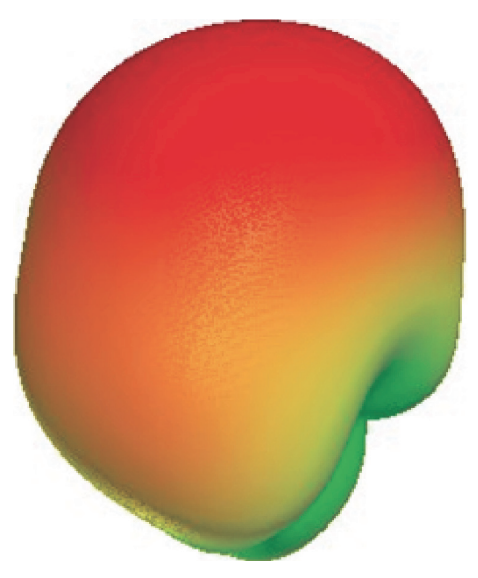

(c)
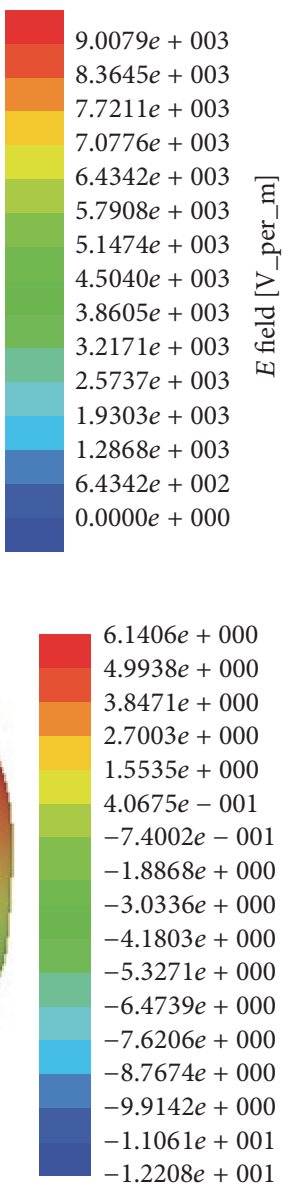

$-1.2208 e+001$



(b)

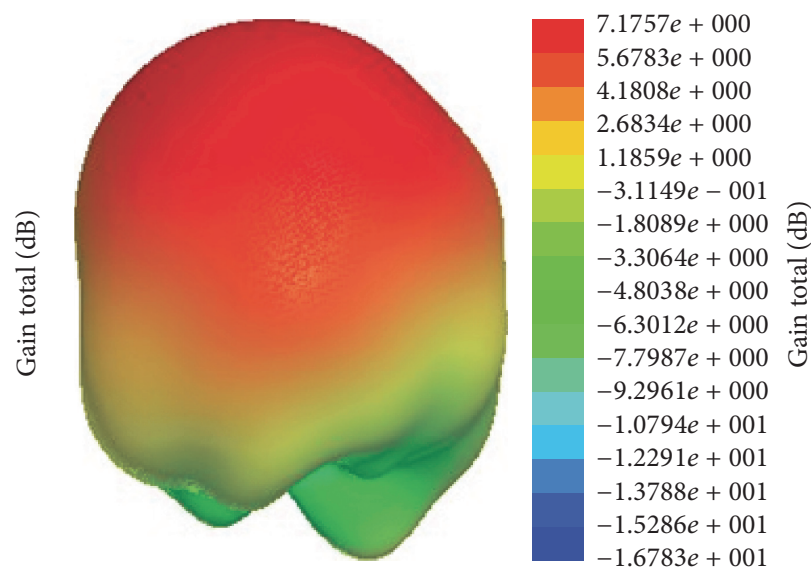

(d)

FIGURE 5: Simulated electric field distributions and 3D radiation patterns. Electric field distribution at (a) $3.5 \mathrm{GHz}$ and (b) $5.25 \mathrm{GHz}$. 3D radiation patterns at (c) $3.5 \mathrm{GHz}$ and (d) $5.25 \mathrm{GHz}$.

which is difficult to be good impedance matches at all operation bands. Here three conditions are adopted to achieve a good antenna performance. Feeding without slot, with one-annular-ring slot corresponding to a capacitance, and with a two-annular-ring slot equal to a shunt capacitance is used to compensate the feeding port, respectively. The input impedance for three conditions is simulated and depicted in Figure 5(a). Obviously, the curve for the antenna feeding with a two-annular-ring slot has the flattest curves compared to that of the other two cases at both bands, indicating an improved impedance matching. For further analysis, the reflection coefficients under the three cases are shown in Figure 5(b). It is clearly observed that the antennas feeding with one-annular-ring slot or without slot shows poor 


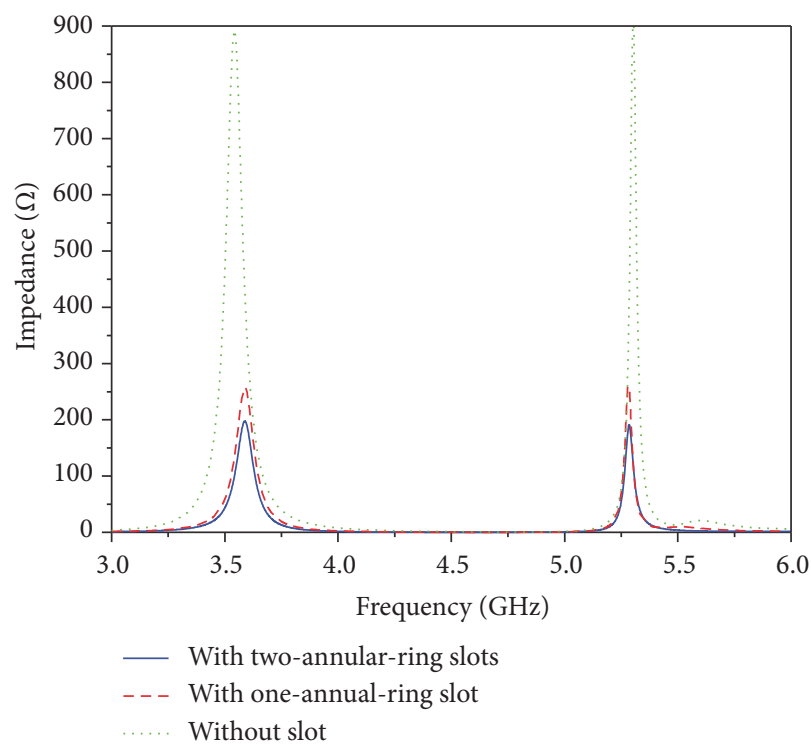

(a)

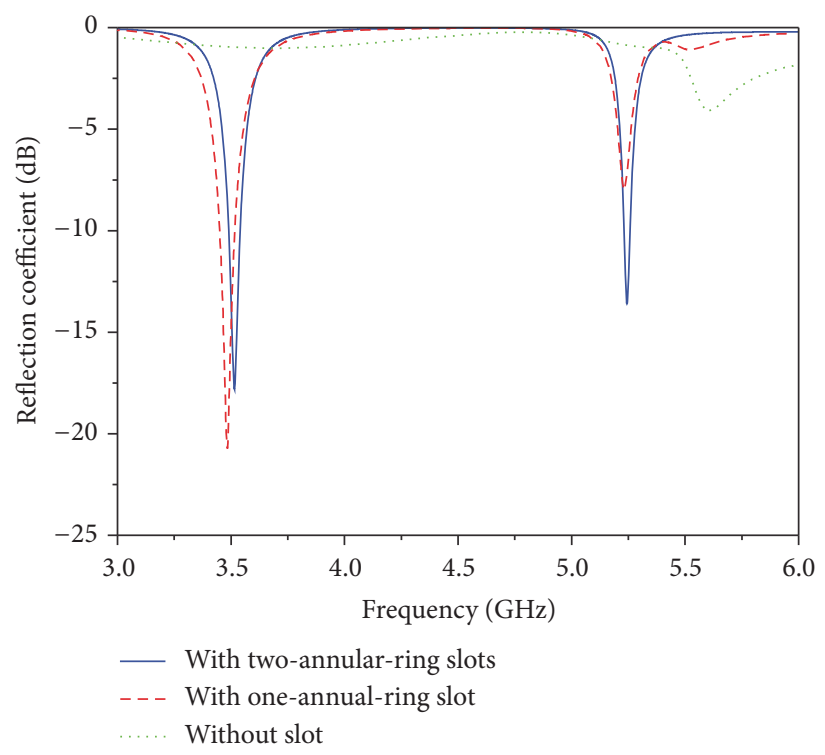

(b)

FIGURE 6: Simulated (a) input impedance and (b) S-parameters feeding with no slot, one-annular-ring slot, and two-annular-ring slot, respectively.

impedance matches, especially for the one feeding without slot, while the return loss is less than $-14.5 \mathrm{~dB}$ for both bands of the antenna feeding with two-annular-ring slot (see Figure 6).

Figure 5 illustrates the simulated electric field distributions and 3D radiation patterns for two interesting modes, respectively. It is observed that the electric fields are $180^{\circ}$ out of phase corresponding to a half wavelength at $f_{-1}=$ $3.5 \mathrm{GHz}$ and $f_{+1}=5.25 \mathrm{GHz}$, respectively. The discrepancy of electric field distribution in the mushroom structures gives rise to different kinds of radiation modes, indicating that the antenna operates with a combination of $\mathrm{RH}$ patch and mushroom parts. As a consequence of similar $180^{\circ}$ out-ofphase electric field distribution, patch-like radiation patterns are expected in Figures 5(c) and 5(d).

For experimental verification, the proposed antenna has been fabricated, assembled, and measured with return loss through a ME7808A vector network analyzer while radiation patterns are through the far-field measurement system in an anechoic chamber. Figure 7 depicts the return loss of the proposed antenna with the photograph displayed in the inset. Obviously, the return loss for the EM simulation and circuit simulation and measurement are in good agreement, indicating the rationality and accuracy of the asymmetrical hybrid resonant circuit model. As the measured result shows, the $-10 \mathrm{~dB}$ impedance bandwidths for both bands are $51 \mathrm{MHz}$ and $32 \mathrm{MHz}$ with the center frequencies located at $3.5 \mathrm{GHz}$ and $5.25 \mathrm{GHz}$, respectively. The bandwidth of the proposed antenna is determined by the $Q$ value of the resonators, which can be enhanced by increasing the antenna size or substrate thickness. The simulated and measured radiation patterns in the two principal planes are shown in Figure 8. As expected,

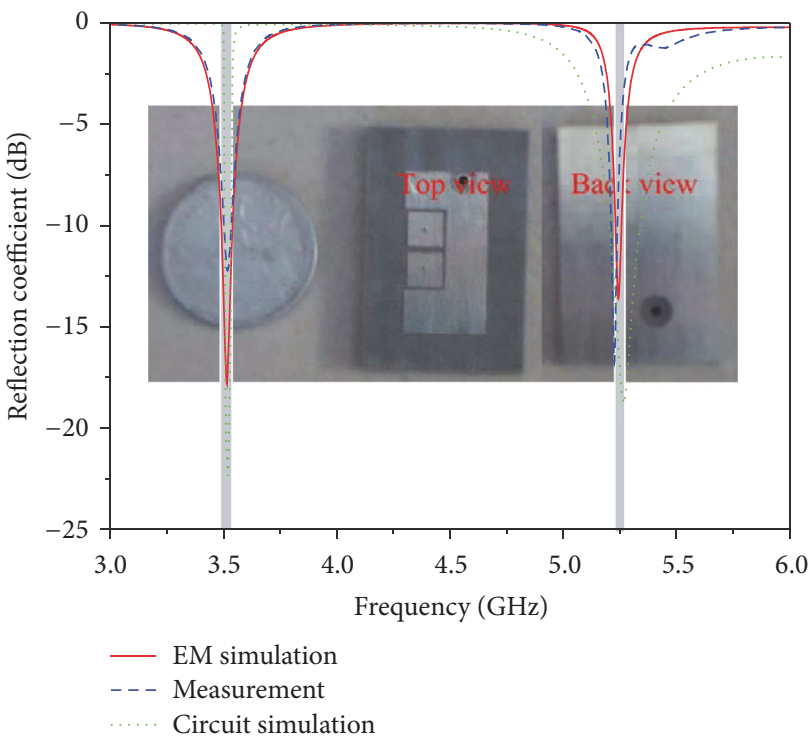

FIGURE 7: Simulated and measured return loss for the dual-band antenna with the photograph displayed in the inset. The shadow represents the $-10 \mathrm{~dB}$ bandwidth of the proposed antenna.

the experimental results are in excellent consistency with the simulated ones with patch-like patterns at both bands. And the measured antenna gain reaches 6.2 and $6.1 \mathrm{~dB}$, respectively. At $3.5 \mathrm{GHz}$, the peak of the cross-polarization is $-10.8 \mathrm{~dB}$ in the E-plane and $-12.6 \mathrm{~dB}$ in the $\mathrm{H}$-plane. And, at $5.25 \mathrm{GHz}$, the values of the cross-polarization are both lower than $-10.3 \mathrm{~dB}$ around the radiation direction. 




(a)



(c)

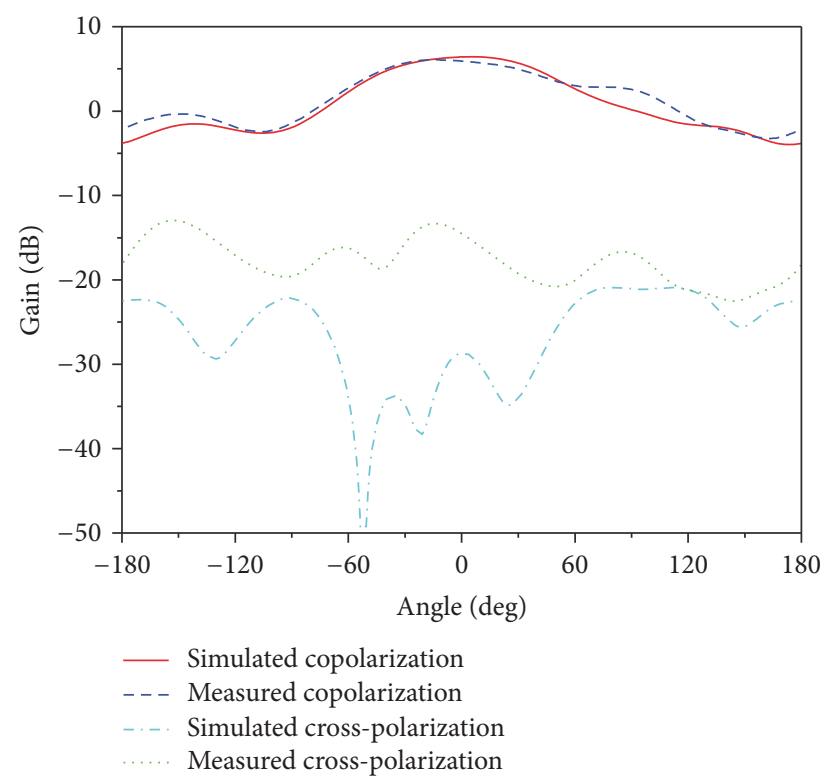

(b)

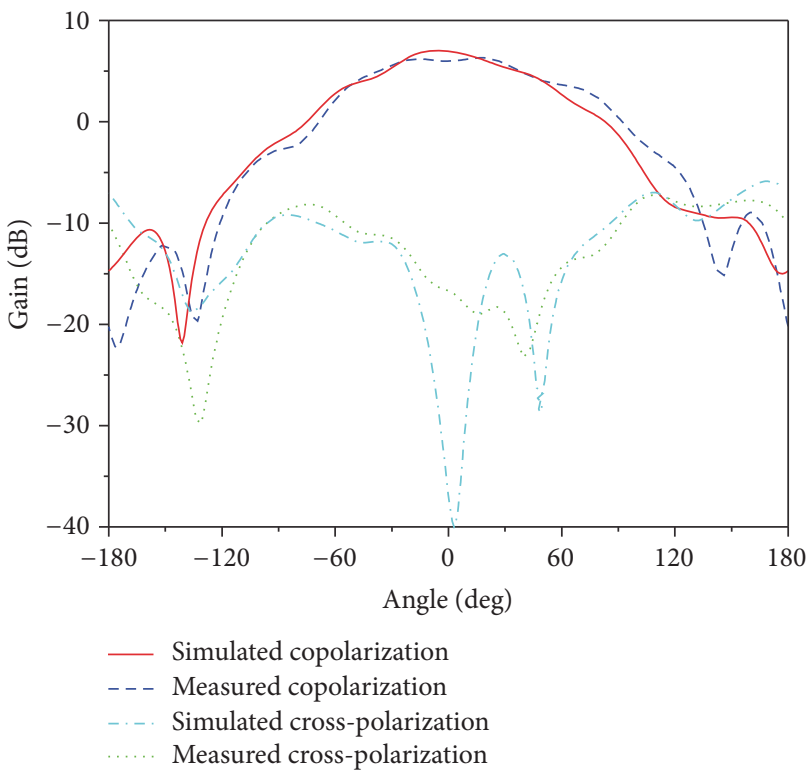

(d)

Figure 8: Radiation patterns of the proposed dual-band antenna. (a) E-plane at $3.5 \mathrm{GHz}$; (b) H-plane at $3.5 \mathrm{GHz}$; (c) E-plane at $5.25 \mathrm{GHz}$; (d) $\mathrm{H}$-plane at $5.25 \mathrm{GHz}$.

\section{Ultrasmall Antenna Design Using Hybrid Resonator}

For further verification and comprehensive analysis of the proposed resonant circuit model, another ultrasmall dualband antenna operating at $f_{0}=2.4 \mathrm{GHz}$ (Bluetooth band with the mode $n=0$ ) and $f_{+1}=3.5 \mathrm{GHz}$ (Wimax band with the mode $n=+1$ ) is designed with the typology shown in Figure 9. We have demonstrated that CSRs are of importance to increase of LH inductance, resulting in an electrical smaller structure and easier method for frequency manipulation. Here CSRs are introduced in the asymmetrical patch antenna with detailed geometrical parameters in Figure 9. The final size of the patch is just $19.8 \times 10 \mathrm{~mm}^{2}$, corresponding to $0.158 \lambda_{0} \times 0.08 \lambda_{0}$, which is smaller than $127 \%$ compared with the available MTM antennas based on mushroom structures $[9,16]$. Also a two-semiannular-ring slot is used for good impedance matches at both bands.

Figure 10 plots the curve of $S_{11}$ for EM simulation, circuit simulation, and measurement. A desirable agreement between the EM simulated and circuit simulated results verifies the rationality of the proposed asymmetrical hybrid resonant circuit model for a second time. Meanwhile a slight frequency blueshift is obtained for the measured result which 


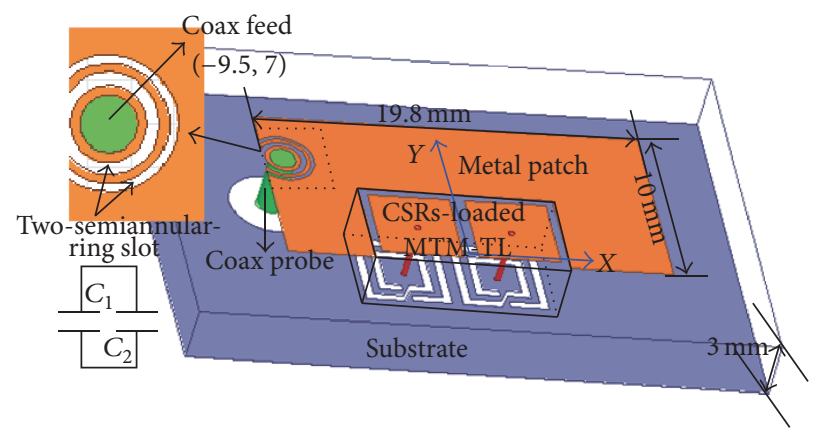

FIGURE 9: Schematics of the proposed antenna with geometrical parameters (in $\mathrm{mm}$ ).

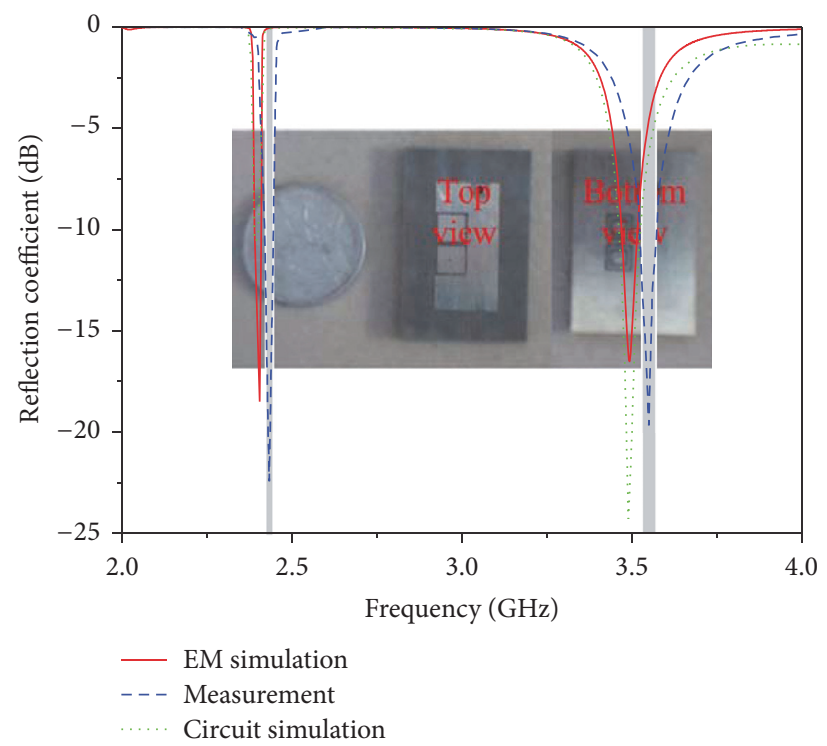

FIGURE 10: Simulated and measured return loss with the photograph of antenna displayed in the inset. The shadow represents the $-10 \mathrm{~dB}$ bandwidth of the proposed antenna.

can be ascribed to the inherent tolerance in the fabrication process. From the measured result, two operating bands show $10 \mathrm{~dB}$ impedance bandwidths of $25 \mathrm{MHz}$ around $f_{0}$ and $50 \mathrm{MHz}$ near $f_{+1}$, corresponding to $1.04 \%$ and $1.43 \%$, respectively.

To extensively analyze the working mechanism of different working modes, the electric field distribution at the patch radiator is shown in Figure 11. Referring to Figure 11(a), the electric field is in phase along the xoy plane in the mode $n=0$ at $f_{0}=2.4 \mathrm{GHz}$, indicating that an infinite wavelength is supported at the ZOR frequency. As appreciated, the omnidirectional 3D radiation pattern is observed in Figure 11(c), when, at the mode $n=+1$, an out-of-phase electric field distribution is obtained along the $x$ direction, indicating a patch-like radiation pattern at $f_{+1}=3.5 \mathrm{GHz}$ shown in Figure 11(d).

The radiation patterns at two special modes are measured in two principle planes with the results shown in Figure 12. According to the radiation patterns, it is obvious that the experimental results are in excellent consistency with the simulated ones. A monopolar and a patch-like patterns are observed as expected in both modes $n=0$ and $n=+1$. A low cross-polarization less than $-15.2 \mathrm{~dB}$ is observed except the one in the H-plane of $n=0$ mode. The cross-polarization level is about $-7.5 \mathrm{~dB}$ lower than the copolarization at around $-90^{\circ}$ and $110^{\circ}$, which is due to the radiation of CSRsloaded mushroom at the edge of the patch. In addition, the antenna exhibits a peak gain of $2.23 \mathrm{~dB}$ and $5.72 \mathrm{~dB}$ for both bands, respectively. The relative lower gain for $n=0$ is due to the omnidirectional radiation pattern in H-plane.

For a deep insight into the working mechanism of the hybrid resonant circuit, a detail comparison of circuit parameters and antenna performances are shown in Table 1. Clearly, the LH inductor consisting of $L_{p}$ and $L_{p 1}$ increases $115.8 \%$ with the insertion of CSRs, resulting in lower working frequencies as well as a compact structure. The coupling between the mushroom and CSRs gives rise to the increasing 
TABLE 1: Comparison of circuit parameters and performances for proposed two antennas.

\begin{tabular}{|c|c|c|c|c|c|c|c|}
\hline \multirow[b]{2}{*}{ Antenna type } & \multicolumn{6}{|c|}{ CRLH part } & \multirow[b]{2}{*}{ Size } \\
\hline & $C_{g}(\mathrm{pF})$ & $L_{s}(\mathrm{nH})$ & $C_{1}(\mathrm{pF})$ & $C_{p}(\mathrm{pF})$ & $L_{p}(\mathrm{nH})$ & $L_{p 1}(\mathrm{nH})$ & \\
\hline I & 0.42 & 2.24 & 0.16 & 1.95 & 1.01 & 0 & $0.21 \lambda_{0} \times 0.08 \lambda_{0}$ \\
\hline II & 0.49 & 4.3 & 0.15 & 1.92 & 0.98 & 1.2 & $0.158 \lambda_{0} \times 0.08 \lambda_{0}$ \\
\hline \multirow{2}{*}{ Antenna type } & \multicolumn{4}{|c|}{ RH part } & $\mathrm{WF}(\mathrm{GHz})$ & BW & Gain (dB) \\
\hline & $C(\mathrm{pF})$ & $C_{R}(\mathrm{pF})$ & $L_{R}(\mathrm{nH})$ & $R(\Omega)$ & WF (GHZ) & $(\mathrm{MHz})$ & \\
\hline I & 0.21 & 5.48 & 1.88 & 3.25 & $3.5 / 5.25$ & $51 / 32$ & $6.1 / 6.2$ \\
\hline II & 0.21 & 5.46 & 1.91 & 3.05 & $2.4 / 3.5$ & $25 / 50$ & $2.23 / 5.72$ \\
\hline
\end{tabular}
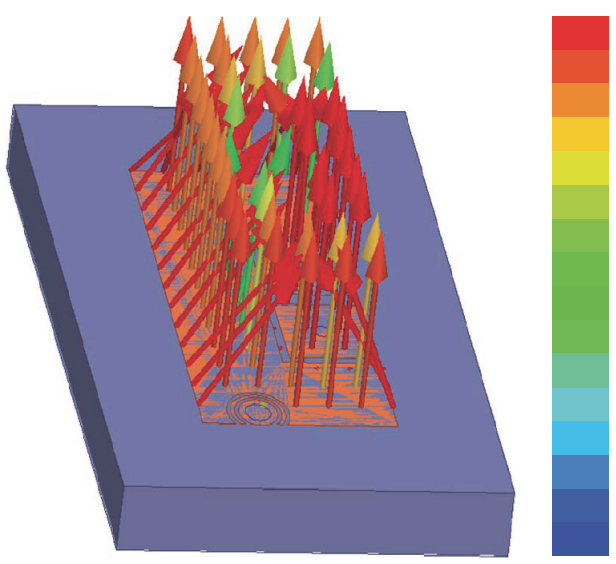

$8.0000 e+003$ $4.9628 e+003$ $3.0787 e+003$ $1.9099 e+003$ $1.1848 e+003$ $7.3497 e+002$ $4.5594 e+002$ $2.8284 e+002$ $1.7546 e+002$ $1.0885 e+002$ $6.7523 e+001$ $4.1888 e+001$ $2.5985 e+001$ $1.6120 e+001$ $1.0000 e+001$

(a)



(c)
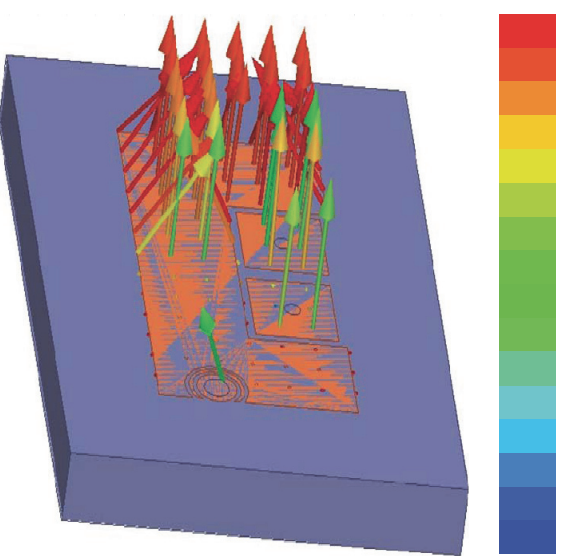

$.0000 e+003$ $4.9628 e+003$ $3.0787 e+003$ $1.9099 e+003$ $1.1848 e+003$ $7.3497 e+002$ $4.5594 e+002$ $2.8284 e+002$ $1.7546 e+002$ $1.0885 e+002$ $6.7523 e+001$ $4.1888 e+001$ $2.5985 e+001$ $1.6120 e+001$ $1.0000 e+001$

(b)

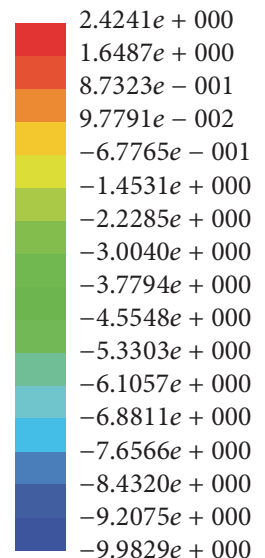

氞

$6.1098 e+000$ $5.0835 e+000$ $4.0572 e+000$ $3.0309 e+000$ $2.0046 e+000$ $9.7826 e-001$ $-4.8035 e-002$ คิ $-1.0743 e+000$ $-2.1006 e+000$ $-3.1269 e+000$ $-4.1532 e+000$ $-5.1795 e+000$ $-6.2058 e+000$ $-7.2321 e+000$ $-8.2584 e+000$ $-9.2847 e+000$ $-1.0311 e+001$

(d)

FIGURE 11: Simulated electric field distributions and 3D radiation patterns. Electric field distribution at (a) 2.4 GHz and (b) 3.5 GHz. 3D radiation patterns at (a) $2.4 \mathrm{GHz}$ and (b) $3.5 \mathrm{GHz}$.

of the parameter values of $L_{s}$ and $C_{g}$. The value of radiation resistance $R$ is larger for antenna II, indicating a higher antenna gain. A similar RH patch structure for the two antennas gives rise to almost the same parameter values of $\mathrm{RH}$ parts. A comparison of the antenna performances has been provided in Table 2. It can be seen clearly that the proposed antennas are electrically smaller compared with these references. Moreover, the measured antenna gain is very comparable with those references $[10,16]$.

\section{Competing Interests}

The authors declare that they have no competing interests. 
TABLE 2: Comparison of antenna performances between the literature and this work.

\begin{tabular}{lcccc}
\hline References & Antenna size $\left(\lambda_{0}\right)$ & Operating frequency $(\mathrm{GHz})$ & Bandwidth $(-10 \mathrm{~dB})$ & Measured gain $(\mathrm{dB})$ \\
\hline$[6]$ & $0.2 \times 0.2 \times 0.017$ & 3.5 & $3.77 \%$ & 4.6 \\
{$[10]$} & $0.177 \times 0.181 \times 0.025$ & 2.58 & $4.60 \%$ & 2.98 \\
{$[11]$} & $0.17 \times 0.33 \times 0.019$ & 3.52 & - & 4.5 \\
{$[12]$} & $0.246 \times 0.246 \times 0.03$ & $1.5 / 2.4 / 3.5$ & $2.63 \% / 3.28 \% / 6.44 \%$ & $4.93 / 2.85 / 5.12$ \\
{$[13]$} & $0.29 \times 0.29 \times 0.06$ & $1.81 / 2.2$ & $-/ \sim 2 \%$ & $4.5 / 6.8$ \\
Pro.I & $0.21 \times 0.08 \times 0.035$ & $3.5 / 5.25$ & $1.46 \% / 0.61 \%$ & $6.2 / 6.1$ \\
Pro.II & $0.158 \times 0.08 \times 0.024$ & $2.4 / 3.5$ & $1.04 \% / 1.43 \%$ & $2.23 / 5.72$ \\
\hline
\end{tabular}

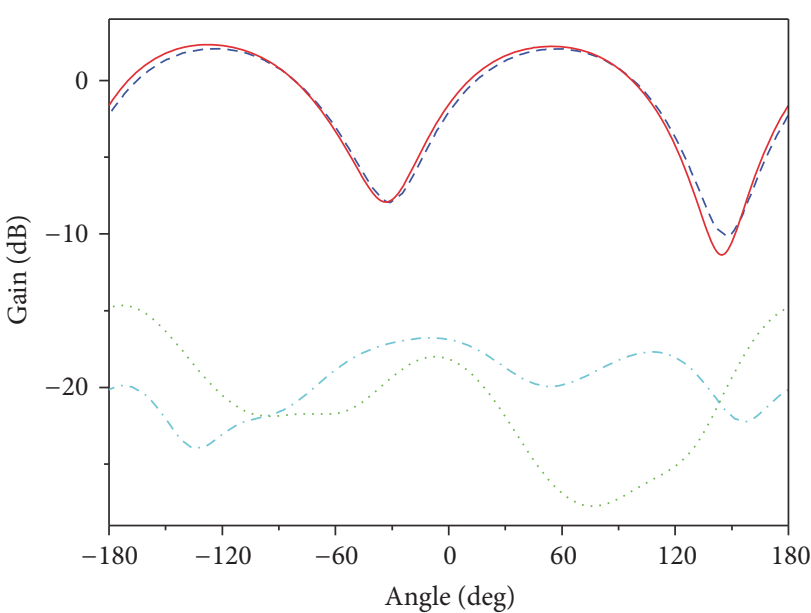

Simulated copolarization

- - - Measured copolarization

..... Simulated cross-polarization

-... Measured cross-polarization

(a)

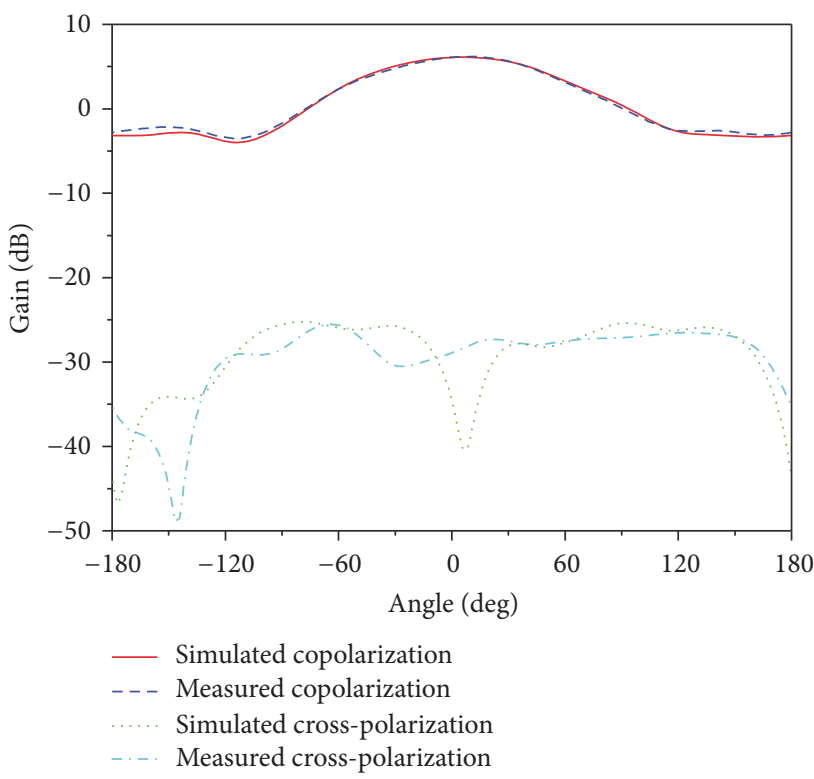

(c)



Simulated copolarization

- - - Measured copolarization

…. Simulated cross-polarization

-... Measured cross-polarization

(b)

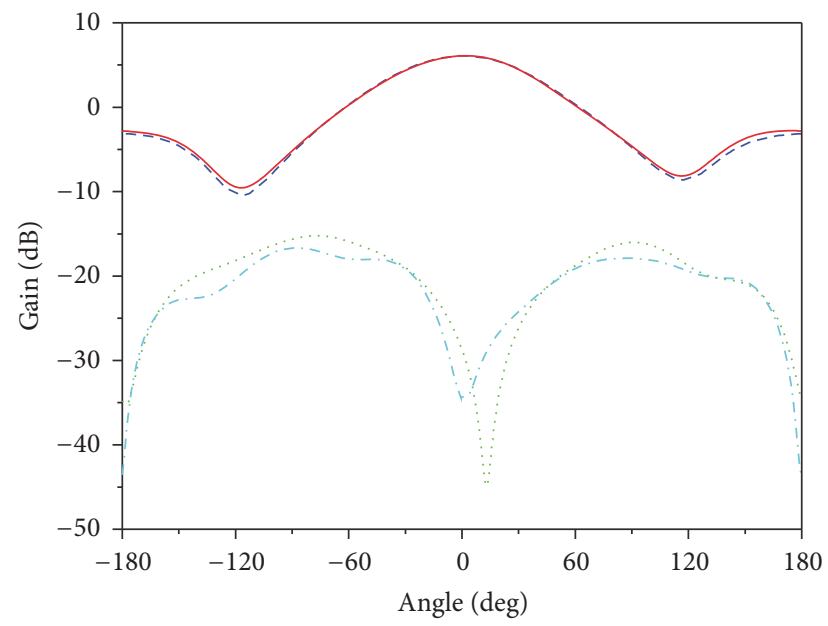

_ Simulated copolarization

- - - Measured copolarization Simulated cross-polarization Measured cross-polarization

(d)

Figure 12: Radiation patterns of the proposed antenna. (a) E-plane at 2.4 GHz; (b) H-plane at 2.4 GHz; (c) E-plane at $3.5 \mathrm{GHz}$; (d) H-plane at $3.5 \mathrm{GHz}$. 


\section{References}

[1] K. U. Ahmed and B. S. Virdee, "Ultra-wideband bandpass filter based on composite right/left handed transmission-line unitcell," IEEE Transactions on Microwave Theory and Techniques, vol. 61, no. 2, pp. 782-788, 2013.

[2] H.-X. Xu, G.-M. Wang, M. Q. Qi, L. Li, and T.-J. Cui, “Threedimensional super lens composed of fractal left-handed materials," Advanced Optical Materials, vol. 1, no. 7, pp. 495-502, 2013.

[3] Z. H. Jiang, M. D. Gregory, and D. H. Werner, "Experimental demonstration of a broadband transformation optics lens for highly directive multibeam emission," Physical Review B, vol. 84, no. 16, Article ID 165111, 2011.

[4] T. Cai, G.-M. Wang, X.-F. Zhang, and J.-P. Shi, "Low-profile compact circularly-polarized antenna based on fractal metasurface and fractal resonator," IEEE Antennas and Wireless Propagation Letters, vol. 14, pp. 1072-1076, 2015.

[5] X. M. Yang, Q. H. Sun, Y. Jing et al., "Increasing the bandwidth of microstrip patch antenna by loading compact artificial magneto-dielectrics," IEEE Transactions on Antennas and Propagation, vol. 59, no. 2, pp. 373-378, 2011.

[6] T. Cai, G.-M. Wang, X.-F. Zhang, Y.-W. Wang, B.-F. Zong, and H.-X. Xu, "Compact microstrip antenna with enhanced bandwidth by loading magneto-electro-dielectric planar waveguided metamaterials," IEEE Transactions on Antennas and Propagation, vol. 63, no. 5, pp. 2306-2311, 2015.

[7] Q. Cheng, W. X. Jiang, and T. J. Cui, "Multi-beam generations at pre-designed directions based on anisotropic zero-index metamaterials," Applied Physics Letters, vol. 99, no. 13, Article ID 131913, 2011.

[8] H. E. A. El-Raouf and S. S. Zaheer, "Design of small planar antennas based on double-layered CRLH metamaterials," Microwave and Optical Technology Letters, vol. 54, no. 10, pp. 2224-2229, 2012.

[9] C.-J. Lee, W. Huang, A. Gummalla, and M. Achour, "Small antennas based on CRLH structures: concept, design, and applications," IEEE Antennas and Propagation Magazine, vol. 53, no. 2, pp. 10-25, 2011.

[10] Y. Dong, H. Toyao, and T. Itoh, "Compact circularly-polarized patch antenna loaded with metamaterial structures," IEEE Transactions on Antennas and Propagation, vol. 59, no. 11, pp. 4329-4333, 2011.

[11] A. Lai, K. M. K. H. Leong, and T. Itoh, "Infinite wavelength resonant antennas with monopolar radiation pattern based on periodic structures," IEEE Transactions on Antennas and Propagation, vol. 55, no. 3, pp. 868-876, 2007.

[12] H.-X. Xu, G.-M. Wang, and M.-Q. Qi, "A miniaturized tripleband metamaterial antenna with radiation pattern selectivity and polarization diversity," Progress in Electromagnetics Research, vol. 137, pp. 275-292, 2013.

[13] F. J. Herraiz-Martínez, V. González-Posadas, L. E. GarciaMunoz, and D. Segovia-Vargas, "Multifrequency and dualmode patch antennas partially filled with left-handed structures," IEEE Transactions on Antennas and Propagation, vol. 56, no. 8, pp. 2527-2539, 2008.

[14] S. Yoo and S. Kahng, "CRLH ZOR antenna of a circular microstrip patch capacitively coupled to a circular shorted ring," Progress in Electromagnetics Research C, vol. 25, pp. 15-26, 2012.

[15] S. Mok, S. Kahng, and Y. Kim, "A wide band metamaterial ZOR antenna of a patch coupled to a ring mushroom," Journal of Electromagnetic Waves and Applications, vol. 26, no. 13, pp. 1667-1674, 2012.
[16] B.-C. Park and J.-H. Lee, "Dual-band omnidirectional circularly polarized antenna using zeroth- and first-order modes," IEEE Antennas and Wireless Propagation Letters, vol. 11, pp. 407-410, 2012.

[17] A. Alù, A. D. Yaghjian, R. A. Shore, and M. G. Silveirinha, "Causality relations in the homogenization of metamaterials," Physical Review B-Condensed Matter and Materials Physics, vol. 84, no. 5, Article ID 054305, 2011. 




\section{Enfincering}
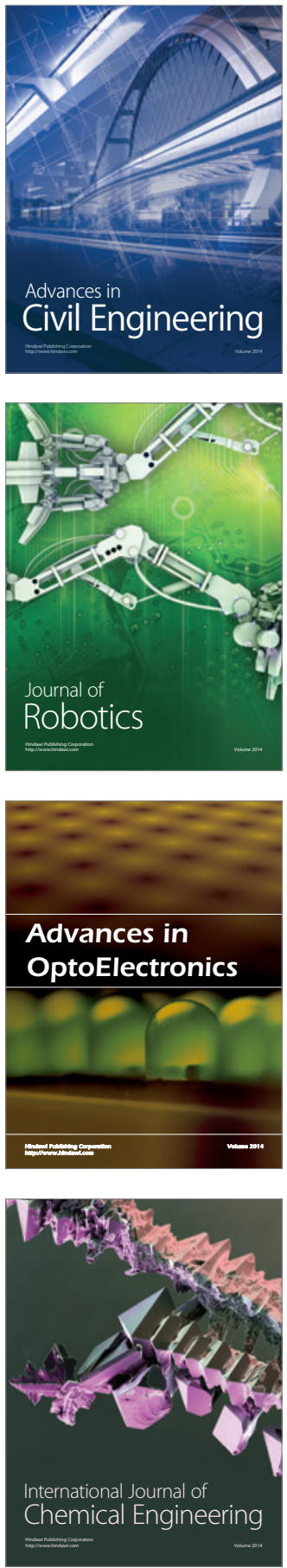

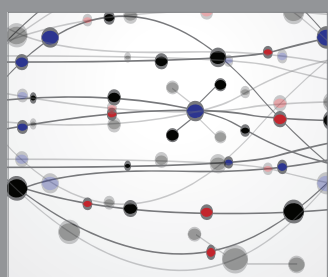

The Scientific World Journal



\section{Hindawi}

Submit your manuscripts at

http://www.hindawi.com
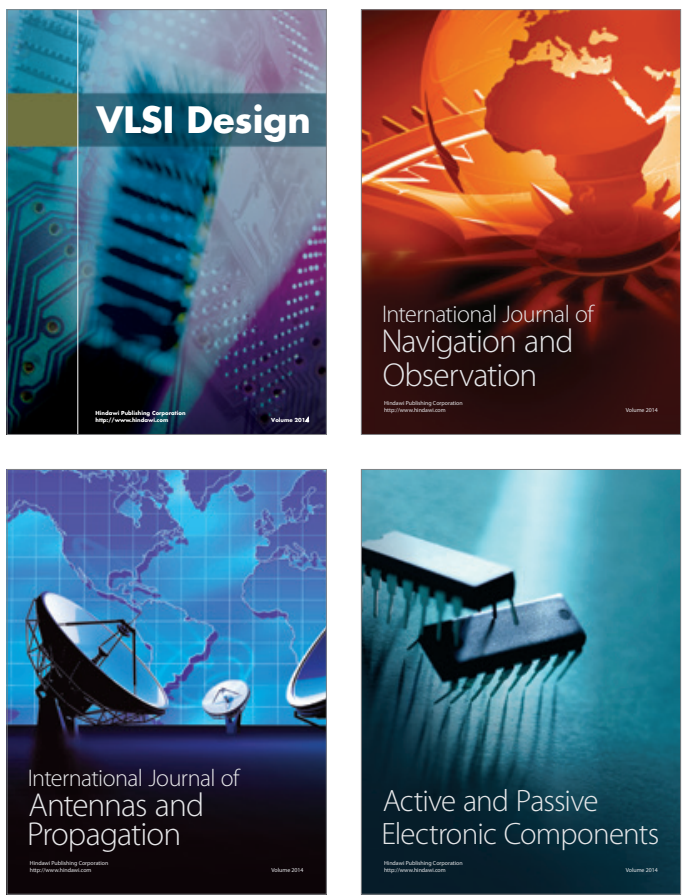
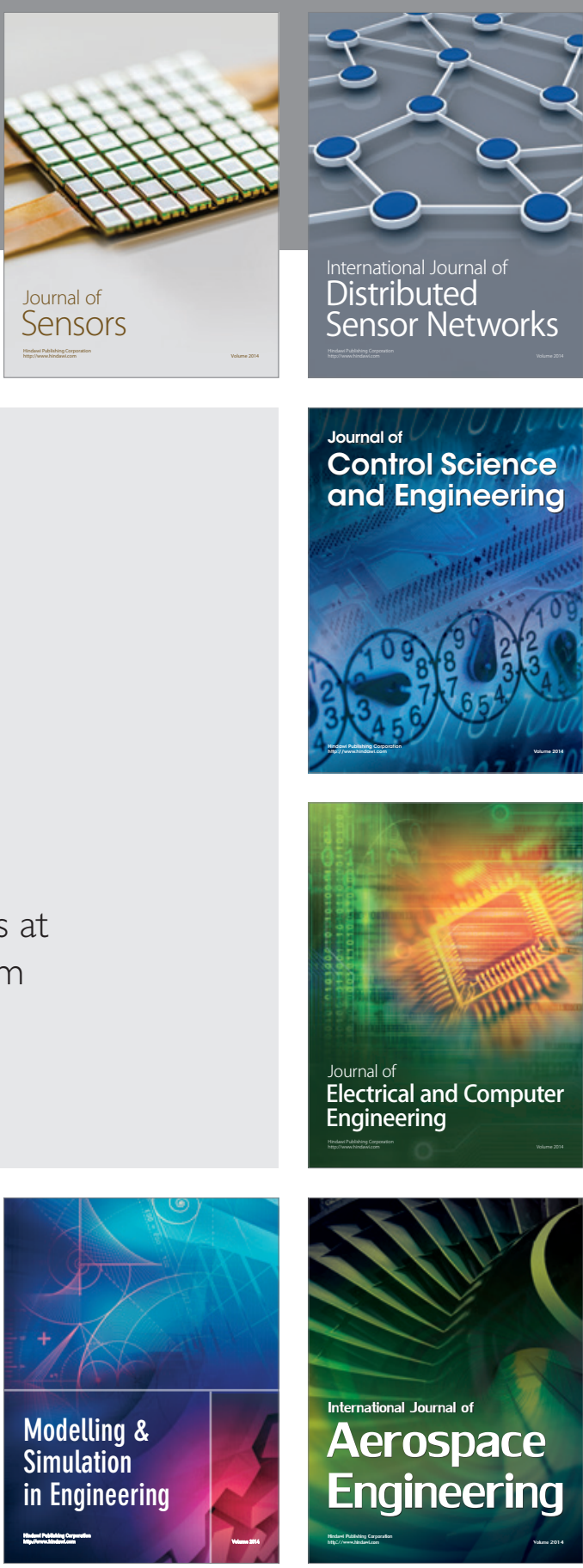

International Journal of

Distributed

Sensor Networks

Journal of

Control Science

and Engineering
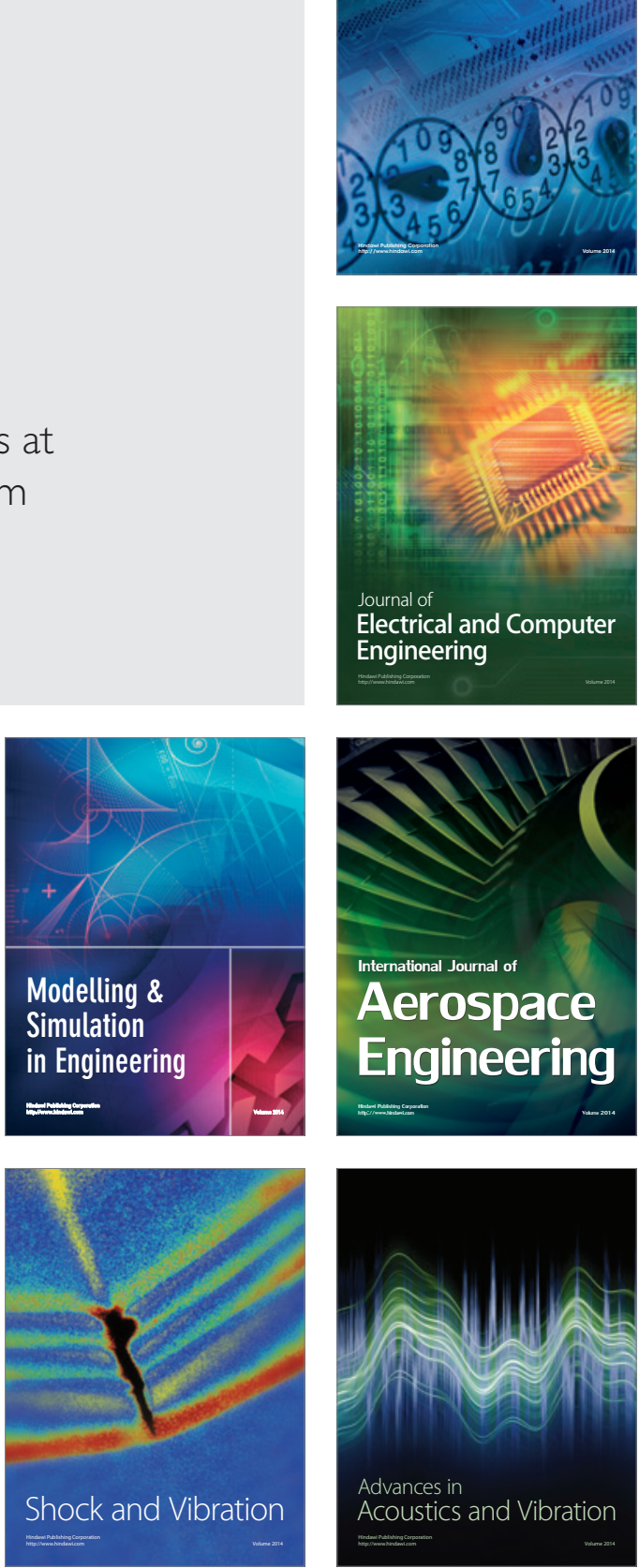